\title{
Synthesis of Homoprotoberberines and 8-Oxoprotoberberines by Sequential Bicyclization of Phenylacetamides
}

\author{
Rafael Suau, ${ }^{*}$ Juan Manuel López-Romero, Antonio Ruiz and Rodrigo Rico \\ Departamento de Química Orgánica, Facultad de Ciencias, Universidad de Málaga, E-29071 Málaga, Spain
}

Received 19 October 1999; revised 22 November 1999; accepted 9 December 1999

\begin{abstract}
The reaction of phenylacetamides with oxalyl chloride/Lewis acid provides a convergent, high-yield entry to C-homoprotoberberine and 8-oxoprotoberberine alkaloids from available starting materials. This approach was used to synthesize 8-oxopseudopalmatine starting from $N$-[ $\beta$ - $\left(3^{\prime}, 4^{\prime}\right.$-dimethoxyphenyl)ethyl]-3,4-dimethoxyphenylacetamide. Some C-homoprotoberberines exhibit significant cytotoxicity against human breast carcinoma cells. (C) 2000 Elsevier Science Ltd. All rights reserved.
\end{abstract}

Protoberberines constitute an important group of isoquinoline alkaloids on account of their physiological activity. ${ }^{1} \mathrm{~A}$ wide array of substituents in the tetracyclic system are known that include 8-oxoprotoberberines (1a,c,d). Some of them were recently isolated from natural sources, ${ }^{2}$ some exhibit antiulcerative properties (8-oxocoptisine, 1d $)^{3}$ and others inhibit the sodium current in human atrial myocytes (8-oxoberberine, 1c). ${ }^{4}$<smiles>[R]c1cc2c(cc1[R])-c1cc3cc([R])c([R4])c([R3])c3c(=O)n1CC2</smiles>

\begin{tabular}{cccccc} 
& $\mathrm{R}_{1}$ & $\mathrm{R}_{2}$ & $\mathrm{R}_{3}$ & $\mathrm{R}_{4}$ & $\mathrm{R}_{5}$ \\
(1a) & $\mathrm{OMe}$ & $\mathrm{OMe}$ & $\mathrm{H}$ & $\mathrm{OMe}$ & $\mathrm{OMe}$ \\
(1b) & $\mathrm{OMe}$ & $\mathrm{OMe}$ & $\mathrm{H}$ & $\mathrm{OMe}$ & $\mathrm{H}$ \\
(1c) & $\mathrm{OCH}_{2} \mathrm{O}$ & $\mathrm{OMe}$ & $\mathrm{OMe}$ & $\mathrm{H}$ \\
(1d) & $\mathrm{OCH}_{2} \mathrm{O}$ & \multicolumn{2}{|c}{$\mathrm{OCH}_{2} \mathrm{O}$} & $\mathrm{H}$
\end{tabular}<smiles>CCC(=O)N1CCc2cc3c(cc2C1=Cc1ccc(OC)c(OC)c1OC)OCO3</smiles>

C-Homoprotoberberines such as hediamine have been isolated from natural sources; ${ }^{5}$ however, these alkaloids can be regarded as artefacts produced during the isolation process rather than as naturally occurring compounds. ${ }^{5 \mathrm{a}}$

From a synthetic point of view, 8-oxoprotoberberines are

Keywords: homoprotoberberines; phenylacetamides; alkaloids; isoquinolines; cytotoxicity; oxalyl chloride.

* Corresponding author. Tel.: +34-5-213-1934; fax: +34-5-213-1941; e-mail: suau@uma.es major intermediates in the synthesis of other protoberberine alkaloids; ${ }^{1 \mathrm{a}, 6}$ various approaches to their total synthesis have been developed. Formation of rings B and $C$ in the final steps is the approach of choice. Ring $\mathrm{B}$ has generally been constructed by variants of the Bischler-Napieralski cyclization $^{1 \mathrm{a}, 7}$ and ring $\mathrm{C}$ in many different ways including: (i) photochemical cyclization of 1-methyleneisoquinolines ${ }^{8}$ (or by Heck reaction) $)^{9}$ or stilbene derivatives; ${ }^{10}$ (ii) reaction of isoquinoline derivatives with arynes, ${ }^{11}$ phthalides, ${ }^{12}$ homophthalic anhydrides ${ }^{13}$ or silyl derivatives; ${ }^{14}$ and (iii) from Reissert compounds. ${ }^{15}$

Recently, the synthesis of 8-oxopseudopalmatine (1a) was accomplished with a $50 \%$ yield by one-pot reaction of the corresponding $N$-ethoxycarbonyl-phenylethylamine with homoveratric acid in polyphosphoric acid. ${ }^{16}$ The simultaneous formation of rings $\mathrm{B}$ and $\mathrm{C}$ has also been accomplished by oxidation of an appropriate 10-membered ring macrolactam with $m$-chloroperbenzoic acid to give the unsubstituted skeleton. ${ }^{17}$

We have developed a new, short synthesis for the cytotoxic isoquinoline alkaloids, 4,5-dioxoaporphines, 3,4-dioxocularines (2) and aristocularines (3) by sequential bicyclization of suitably substituted arylacetamides promoted by oxalyl chloride and tin(IV) chloride. ${ }^{18}$ In this cyclization, oxalyl chloride generates the 2-chloro-oxazolidine-4,5dione (I) precursor of the electrophilic $N$-acyliminium ion and acts as an $\alpha$-dicarbonyl or monocarbonyl transfer agent (Scheme 1).

We envisaged that this approach could be applied to the cyclization of phenylethyl phenylacetamides (Scheme 2) to provide a direct access to ring $\mathrm{C}$-homoprotoberberines and 8-oxoprotoberberines provided decarbonylation occurred under the prevailing reaction conditions. 


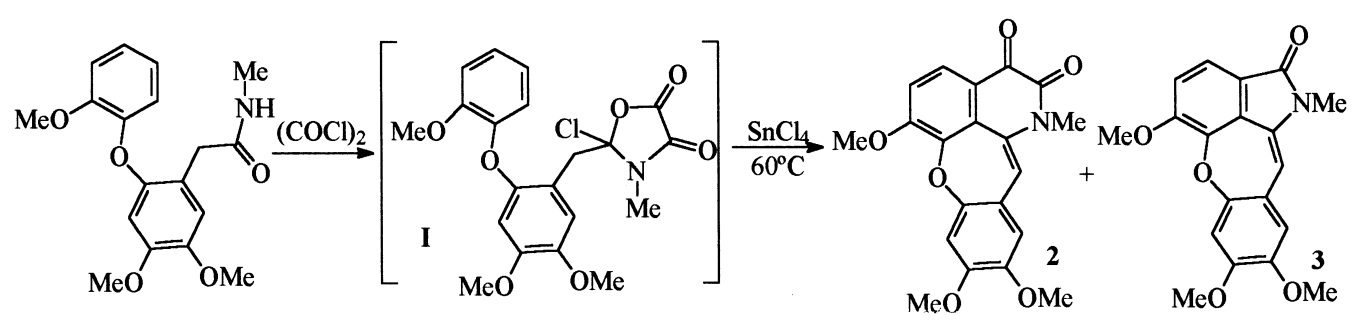

Scheme 1.
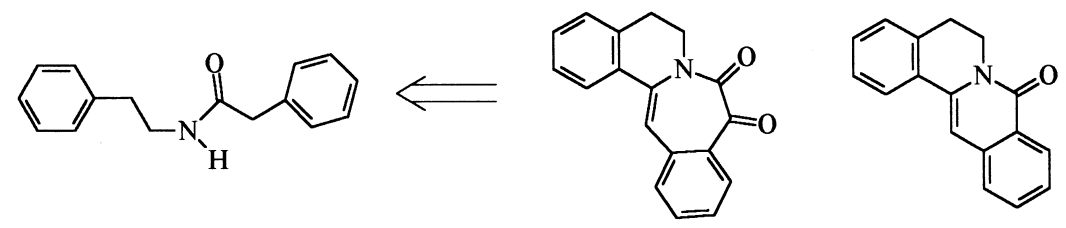

Scheme 2.

This paper describes in detail the reaction of various $N$-[ $\beta$-(phenyl)ethyl]-phenylacetamides with oxalyl chloride/ Lewis acid to afford ring C-homoprotoberberines in a single step by sequential formation of rings $\mathrm{B}$ and $\mathrm{C}$. We also report a straightforward synthesis for 8-oxoprotoberberines from ring C-homoprotoberberines by either thermal decarbonylation or decarboxylation after benzylic acid type of rearrangement.

\section{Results and Discussion}

According to Larsen, ${ }^{19}$ the reaction of (1,2-diphenylethyl)phenylacetamide (4) with oxalyl chloride and $\mathrm{FeCl}_{3}$, in a modified Bischler-Napieralski process, gives the oxalyl adduct 5 in 55\% yield. Acid hydrolysis of this oxazolidindione affords the corresponding 3,4-dihydroisoquinoline (6) (Scheme 3).

In our study, and under similar conditions $\left[(\mathrm{COCl})_{2} / \mathrm{SnCl}_{4}\right.$ at room temperature], the amide 7a gave the related oxazolidindione $8 \mathbf{a}$ in a $63 \%$ yield. However, we knew from previous work that this type of oxalyl adducts is readily opened if a proton can be eliminated from a benzylic position by increasing the reaction temperature. Thus, when the reaction of $7 \mathbf{a}$ was carried out at $60^{\circ} \mathrm{C}$ ( 2 equiv. of $\mathrm{SnCl}_{4}$ ), the yield of $\mathbf{8 a}$ decreased to $8 \%$. At this temperature, and in the presence of $\mathrm{BF}_{3} \cdot \mathrm{OEt}_{2}$ as Lewis acid, opening of the oxazolidinedione and a second cyclization easily occur and provide an almost quantitative yield of the expected C-homoprotoberberine (9a). FAB-MS was consistent with the proposed homoprotoberberine structure, 9a, and exhibited two carbonyl signals and four uncoupled aromatic protons in the CMR and the PMR spectrum, respectively (Scheme 4).

With a less active aromatic ring $\mathrm{D}(\mathbf{7 b})$, the yield of homoprotoberberine (9b) was lower $(65 \%)$. When the aromatic portion of the phenylacetic acid moiety is unsubstituted (7c), the second cyclization faces difficulties and the oxazolidinedione $8 \mathrm{c}$ is obtained (16\%) together with 9c $(56 \%)$. When ring $\mathrm{A}$ is not activated (7d), the yield of the corresponding homoprotoberberine, 9d, drops to the $23 \%$, the benzylidene isoquinoline $\mathbf{1 0}$ being the second reaction product isolated.

Under these conditions $\left(60^{\circ} \mathrm{C}\right)$ and with $\mathrm{SnCl}_{4}$ as Lewis acid, the reaction of amide $\mathbf{7 a}$ afforded a quantitative yield of a crystalline precipitate that was characterized as a complex of the homoprotoberberine retaining one molecule of catalyst $\left(\mathbf{9} \cdot \mathbf{S n C l} \mathbf{2}_{2}\right)$. An XPS study revealed the presence of $\mathrm{Sn}(\mathrm{IV})$, and a 1:1:2 proportion of $\mathrm{N} / \mathrm{Sn} / \mathrm{Cl}$, and suggested that the metal must be coordinated to the carbonyls of the $\alpha$-oxoamide functionality. The complex was exhaustively treated with diluted $\mathrm{NaOH}$ to remove the metal, and 9a was obtained in a $29 \%$ yield. Similar results were achieved with $\mathrm{TiCl}_{4}$, the complex $\mathbf{9 a} \cdot \mathbf{T i C l}_{\mathbf{2}}$ being isolated and identified (XPS).

In the reaction of $\mathbf{7 b}$ with $\mathrm{SnCl}_{4}$, the second cyclization is slower and the oxazolidine dione $\mathbf{8 b}$ and the pyrazoloisoquinoline dione $\mathbf{1 1}$ are isolated in low yields, in addition to the expected complex $\left(\mathbf{9 b} \cdot \mathbf{S n C l} \mathbf{l}_{\mathbf{2}}\right)$.

Decarbonylation of 9a was accomplished via a benzylic acid type rearrangement ${ }^{20}$ followed by oxidative decarboxylation, a reaction that has previously been successfully

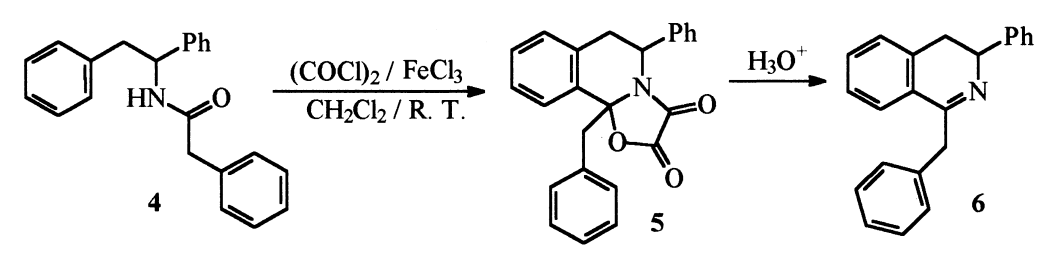




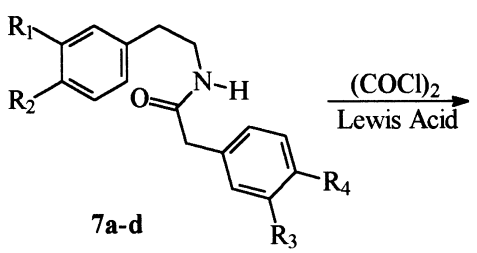

a) $\mathrm{R}_{1}=\mathrm{R}_{2}=\mathrm{R}_{3}=\mathrm{R}_{4}=\mathrm{OMe}$

b) $\mathrm{R}_{1}=\mathrm{R}_{2}=\mathrm{R}_{4}=\mathrm{OMe} ; \mathrm{R}_{3}=\mathrm{H}$<smiles>[R]c1ccc(C[C@]23OC(=O)C(=O)N2CCc2cc([R])c([R])cc23)cc1[R]</smiles><smiles>[R]c1cc2c(cc1[R])-c1cc3cc([R])c([R])cc3c(=O)n1CC2</smiles>

c) $\mathrm{R}_{1}=\mathrm{R}_{2}=\mathrm{OMe} ; \mathrm{R}_{3}=\mathrm{R}_{4}=\mathrm{H}$

d) $\mathrm{R}_{1}=\mathrm{R}_{2}=\mathrm{H} ; \mathrm{R}_{3}=\mathrm{R}_{4}=\mathrm{OMe}$<smiles>COc1ccc(/C=C2\c3ccccc3CCN2C(=O)C(=O)O)cc1OC</smiles><smiles>COc1ccc(C2=C3c4cc(OC)c(OC)cc4CCN3C(=O)C2=O)cc1</smiles>

Scheme 4.

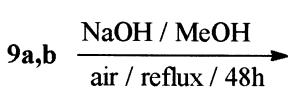

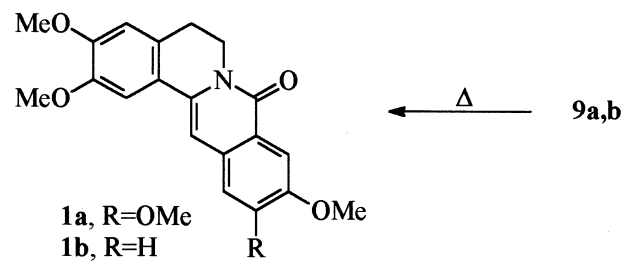

Scheme 5.

applied to related $\alpha$-oxoamides. Thus, treatment of $\mathbf{9 a}$ with $\mathrm{NaOH}$ in methanol afforded 8-oxopseudopalmatine (1a) (Scheme 5).

Gas evolution was observed on heating over $170^{\circ} \mathrm{C}$ while the melting point of 9a was determined. TGA analyses revealed an exothermic peak at $175^{\circ} \mathrm{C}$, with a weight lost of about $7 \%$ that corresponds to $\mathrm{CO}$ elimination. Under these conditions, 9a was decarbonylated to $\mathbf{1 a}$ (70\%). Similar results were obtained for $\mathbf{9 b}$. On heating this compound at $155^{\circ} \mathrm{C}, 11$-demethoxy-8-oxopseudopalmatine (1b) was obtained.

The potency of the C-homoprotoberberines $(9 \mathbf{a}-\mathbf{d})$ as cytotoxic agents was tested against MDA-MB-231 human breast carcinoma cells. Compounds $9 \mathbf{a}$ and $9 \mathbf{c}$ exhibited significant activity $\left(\mathrm{IC}_{50}: 5.2\right.$ and $6.2 \mu \mathrm{g} / \mathrm{mL}$, respectively; $48 \mathrm{~h}$ treatment $),{ }^{21}$ while 1 a was found to be inactive. The consistent cytotoxicity found for the $\mathrm{C}$-homoprotoberberines and related dioxoisoquinolines suggests that they may function as antineoplastic agents.

\section{Experimental}

\section{General methods}

The melting points were determined on a Gallenkamp instrument and are given uncorrected. UV spectra were recorded on a Hewlett-Packard 8452A spectrophotometer and IR spectra on a Perkin-Elmer 883 spectrophotometer.
Mass spectra were recorded on a HP-MS 5988A spectrometer operating at $70 \mathrm{eV}$. NMR spectra were obtained on a Bruker WP-200 SY instrument at $200 \mathrm{MHz}$ for ${ }^{1} \mathrm{H}$ and $50.3 \mathrm{MHz}$ for ${ }^{13} \mathrm{C}$. ${ }^{1} \mathrm{H}$ Chemical shifts $\left(\delta_{\mathrm{H}}\right)$ are given relative to residual $\mathrm{CHCl}_{3}\left(\delta_{\mathrm{H}} 7.24 \mathrm{ppm}\right)$ in deuteriochloroform or to residual $\mathrm{CHD}_{2} \mathrm{SOCD}_{3}$ in DMSO- $d_{6}\left(\delta_{\mathrm{H}} 2.50 \mathrm{ppm}\right) . J$ values are in Hertz. ${ }^{13} \mathrm{C}$ Chemical shifts $\left(\delta_{\mathrm{C}}\right)$ are given relative to $\mathrm{CDCl}_{3}$ $\left(\delta_{\mathrm{C}} 77.0 \mathrm{ppm}\right)$ in deuteriochloroform or $\mathrm{CD}_{3} \mathrm{SOCD}_{3}\left(\delta_{\mathrm{C}}\right.$ $39.7 \mathrm{ppm}$ ) in DMSO- $d_{6}$. X-Ray Photoelectron Spectra (XPS) were obtained using a Physical Electronics PHI 5700 spectrometer, and TGA analyses were conducted on a RigakuTermoflex apparatus. TLC analyses were performed on silica gel 60 F 256 plates, and column chromatography was carried out on silica gel 60 (70-230 mesh). Amides 7a-d were prepared by condensation of phenylethyl amine and homoveratrylamine with the corresponding phenylacetic acids.

\section{Reaction of amides $7 \mathrm{a}-\mathrm{d}$ with $(\mathrm{COCl})_{2} / \mathrm{BF}_{3} \cdot \mathrm{OEt}_{2}$. General procedure}

To a degassed (Ar) solution of the amide $7 \mathbf{a}-\mathbf{d}(2.6 \mathrm{mmol})$ in dry dichloromethane $(25 \mathrm{~mL})$, in a septum sealed round bottom flask, oxalyl chloride $(6 \mathrm{~mL}, 26 \mathrm{mmol})$ was added. The solution was stirred at $20^{\circ} \mathrm{C}$ for $15 \mathrm{~min}$, followed by addition of $\mathrm{BF}_{3} \cdot \mathrm{OEt}_{2}(1.37 \mathrm{~mL}, 13 \mathrm{mmol})$ via a syringe. The mixture was stirred at $60^{\circ} \mathrm{C}$ for $24 \mathrm{~h}$, time during which a solid precipitated. The solid was filtered, washed with $\mathrm{CH}_{2} \mathrm{Cl}_{2}$ and dried to afford the $\mathrm{C}$-homoprotoberberines $\mathbf{9 a}-\mathbf{d}$. The filtrates were washed with water, dried over $\mathrm{MgSO}_{4}$ and concentrated in vacuo. The residue was purified by column chromatography to give the stated products. 
Reaction of $N$-[2-(3,4-dimethoxyphenyl)ethyl]-3,4-dimethoxyphenylacetamide (7a)

The yellow solid ( $1 \mathrm{~g}, 98 \%$ ) was recrystallized from $\mathrm{CH}_{2} \mathrm{Cl}_{2}$ and identified as 9a. No additional products were isolated from the filtrates.

2,3,11,12-Tetramethoxy-5,6-dihydroisoquino[1,2-b][3]benzazepine-8,9-dione (9a). Dark yellow plates, mp 168$170^{\circ} \mathrm{C}\left(\mathrm{CH}_{2} \mathrm{Cl}_{2}\right)$ (gas evolution); $\nu(\mathrm{KBr}) \mathrm{cm}^{-1} 1639,1604$; $\lambda_{\max }(\mathrm{EtOH}) \mathrm{nm}(\log \epsilon): 380$ (3.26), 340 (3.64), 288 (4.00), $242(3.67) ; \delta_{\mathrm{H}}\left(\mathrm{DMSO}-d_{6}\right) 8.55$ (s, 1H, H-1), 7.59 (s, 1H, Ar-H), 7.51 (s, 1H, Ar-H), 7.34 (s, 1H, H-4), 7.04 (s, 1H, H14), $4.62\left(\mathrm{brt}, 2 \mathrm{H}, \mathrm{CH}_{2} \mathrm{~N}\right), 4.05\left(\mathrm{~s}, 3 \mathrm{H}, \mathrm{OCH}_{3}\right), 4.93(\mathrm{~s}, 3 \mathrm{H}$, $\left.\mathrm{OCH}_{3}\right), 3.90\left(\mathrm{~s}, 3 \mathrm{H}, \mathrm{OCH}_{3}\right), 3.84\left(\mathrm{~s}, 3 \mathrm{H}, \mathrm{OCH}_{3}\right), 3.12$ (br t, $\left.2 \mathrm{H}, \mathrm{CH}_{2}\right) ; \delta_{\mathrm{C}}\left(\mathrm{CDCl}_{3}+\mathrm{TFA}\right) 164.5,159.0(\mathrm{C}=\mathrm{O}), 153.8$, $152.4,149.0,146.9\left(C-\mathrm{OCH}_{3}\right), 139.1,138.3,129.1$, $119.7,118.7$ (C), 119.2, 111.0, 109.0, 105.1, $104.7(\mathrm{CH})$, $57.2\left(\mathrm{OCH}_{3}\right), 56.8\left(\mathrm{OCH}_{3}\right), 56.6\left(\mathrm{OCH}_{3}\right), 56.2\left(\mathrm{OCH}_{3}\right), 52.8$ $\left(\mathrm{CH}_{2} \mathrm{~N}\right), 26.8\left(\mathrm{CH}_{2}\right) ; \mathrm{m} / \mathrm{z}(\%) 395\left(\mathrm{M}^{+}, 0\right), 367(9), 352$ (100), 336 (36); m/z FAB-MS (\%) $396\left(\mathrm{MH}^{+}\right.$, 45); Anal. Calcd for $\mathrm{C}_{22} \mathrm{H}_{21} \mathrm{NO}_{6}$ : C 66.83, H 5.35, N 3.54\%, found $\mathrm{C}$ 66.68, H 5.33 , N 3.82 .

\section{Reaction of $N$-[2-(3,4-dimethoxyphenyl)ethyl $] 4-m e t h-$ oxyphenylacetamide (7b)}

The yellow solid $(0.61 \mathrm{~g}, 65 \%)$ was identified as $9 \mathbf{b}$. No additional products were isolated from the filtrates.

2,3,12-Trimethoxy-5,6-dihydroisoquino[1,2-b][3]benzazepine-8,9-dione (9b). Bright yellow plates, $\mathrm{mp} 150-151^{\circ} \mathrm{C}$ $\left(\mathrm{CH}_{2} \mathrm{Cl}_{2}\right)$ (gas evolution); $\nu(\mathrm{KBr}) \mathrm{cm}^{-1} 1617,1522 ; \lambda_{\max }$ $(\mathrm{EtOH}) \mathrm{nm}(\log \epsilon): 406$ (3.48), 338 (4.08), 276 sh (4.00), 260 (4.09), 230 (4.10), $206(4.01) ; \delta_{\mathrm{H}}\left(\mathrm{CDCl}_{3}+\mathrm{CD}_{3} \mathrm{OD}\right)$ $8.00(\mathrm{~s}, 1 \mathrm{H}, \mathrm{H}-1), 7.82(\mathrm{~d}, 1 \mathrm{H}, J=8.9 \mathrm{~Hz}, \mathrm{H}-13), 7.62(\mathrm{~d}$, $1 \mathrm{H}, J=2.2 \mathrm{~Hz}, \mathrm{H}-10), 7.53$ (dd, $1 \mathrm{H}, J=8.9,2.2 \mathrm{~Hz}, \mathrm{H}-12$ ), 7.27 (s, 1H, H-4), 6.78 (s, 1H, H-14), 4.84 (t, $2 \mathrm{H}, J=6.2 \mathrm{~Hz}$, $\left.\mathrm{CH}_{2} \mathrm{~N}\right), 4.00\left(\mathrm{~s}, 3 \mathrm{H}, \mathrm{OCH}_{3}\right), 3.95\left(\mathrm{~s}, 6 \mathrm{H}, 2 \times \mathrm{OCH}_{3}\right), 3.14(\mathrm{t}$, $\left.2 \mathrm{H}, J=6.2 \mathrm{~Hz}, \mathrm{CH}_{2}\right) ; \delta_{\mathrm{C}}\left(\mathrm{CDCl}_{3}+\mathrm{CD}_{3} \mathrm{OD}\right) 162.7,160.5$ $(\mathrm{C}=\mathrm{O}), 158.5,151.9,149.3\left(\mathrm{C}-\mathrm{OCH}_{3}\right), 136.4,134.3$, 128.0, 124.5, 119.5 (C), 129.9, 128.1, 116.9, 110.5, 107.9, $107.5(\mathrm{CH}), 56.4\left(\mathrm{OCH}_{3}\right), 56.2\left(\mathrm{OCH}_{3}\right), 56.1\left(\mathrm{OCH}_{3}\right), 52.2$ $\left(\mathrm{CH}_{2} \mathrm{~N}\right), 29.6\left(\mathrm{CH}_{2}\right) ; \mathrm{m} / \mathrm{z}(\%) 365\left(\mathrm{M}^{+}, 0\right), 337(2), 322$ (100), $\mathrm{m} / z$ FAB-MS (\%) $366\left(\mathrm{MH}^{+}, 20\right)$; Anal. Calcd for $\mathrm{C}_{21} \mathrm{H}_{19} \mathrm{NO}_{5}$ : C 69.03, H 5.24, N 3.83\%, found C 69.10, $\mathrm{H}$ $5.25, \mathrm{~N} 3.90$.

\section{Reaction of $N$-[2-(3,4-dimethoxyphenyl)ethyl]phenyl- acetamide $(7 \mathrm{c})$}

The yellow solid $(0.49 \mathrm{~g}, 56 \%)$ was identified as $9 \mathrm{c}$. The residue from the filtrates was purified by column chromatography $\left(\mathrm{SiO}_{2}, \mathrm{CH}_{2} \mathrm{Cl}_{2} / \mathrm{CH}_{3} \mathrm{OH}\right.$ 20:0.2) to obtain 8c $(0.15 \mathrm{~g}$, $16 \%)$.

2,3-Dimethoxy-5,6-dihydroisoquino[1,2-b][3]benzazepine8,9-dione (9c). Yellow crystals, mp $260-261^{\circ} \mathrm{C}(\mathrm{EtOH})$ (gas evolution); $\nu(\mathrm{KBr}) \mathrm{cm}^{-1} 1636,1602 ; \lambda_{\max }(\mathrm{EtOH})$ $\mathrm{nm}(\log \epsilon): 388$ (3.46), 336 (3.95), 270 (3.88), 252 (4.02), $232(4.07), 210 \mathrm{sh}(2.92) ; \delta_{\mathrm{H}}\left(\mathrm{CDCl}_{3}\right) 8.51(\mathrm{~d}, 1 \mathrm{H}$, $J=8.4 \mathrm{~Hz}, \mathrm{H}-10), 8.06$ (s, 1H, H-1), 7.92 (m, 2H, Ar-H), 7.71 (m, 1H, Ar-H), 7.32 (s, 1H, H-4), 6.80 (s, 1H, H-14), $4.88\left(\mathrm{t}, 2 \mathrm{H}, J=6.3 \mathrm{~Hz}, \mathrm{CH}_{2} \mathrm{~N}\right), 4.01\left(\mathrm{~s}, 3 \mathrm{H}, \mathrm{OCH}_{3}\right), 3.95(\mathrm{~s}$, $\left.3 \mathrm{H}, \mathrm{OCH}_{3}\right), 3.16\left(\mathrm{t}, 2 \mathrm{H}, J=6.3 \mathrm{~Hz}, \mathrm{CH}_{2}\right) ; \delta_{\mathrm{C}}\left(\mathrm{CDCl}_{3}\right) 162.0$, $161.3(\mathrm{C}=\mathrm{O}), 152.3,149.5\left(\mathrm{C}-\mathrm{OCH}_{3}\right), 138.4,138.1,128.4$, 123.0, 119.4 (C), 136.1, 131.8, 129.9, 126.5, 116.9, 110.6, $108.2(\mathrm{CH}), 56.5\left(\mathrm{OCH}_{3}\right), 56.2\left(\mathrm{OCH}_{3}\right), 52.1\left(\mathrm{CH}_{2} \mathrm{~N}\right), 27.1$ $\left(\mathrm{CH}_{2}\right) ; \mathrm{m} / \mathrm{z}(\%) 335\left(\mathrm{M}^{+}, 0\right), 307$ (1), 292 (25), 276 (12), 212 (39), 170 (100); m/z FAB-MS (\%) $336\left(\mathrm{MH}^{+}, 10\right)$; Anal. Calcd for $\mathrm{C}_{20} \mathrm{H}_{17} \mathrm{NO}_{4}$ : C 71.63, H 5.11, N 4.18\%, found C 72.04, H 5.18, N 4.32.

10b-Benzyl-6,10b-dihydro-8,9-dimethoxy-5H-oxazolo[2,3-a] isoquinoline-2,3-dione (8c). White amorphous solid, mp $152-155^{\circ} \mathrm{C}\left(\mathrm{CH}_{2} \mathrm{Cl}_{2} / \mathrm{CH}_{3} \mathrm{OH}\right)$ (gas evolution); $\nu(\mathrm{KBr}$ ) $\mathrm{cm}^{-1} 1807,1734,1608 ; \lambda_{\max }\left(\mathrm{CHCl}_{3}\right) \mathrm{nm}(\log \epsilon): 286$ (3.42), $244(3.88) ; \delta_{\mathrm{H}}\left(\mathrm{CDCl}_{3}\right) 7.27(\mathrm{~m}, 3 \mathrm{H}, \mathrm{Ar}-\mathrm{H}), 7.08$ $(\mathrm{m}, 2 \mathrm{H}, \operatorname{Ar}-\mathrm{H}), 6.88(\mathrm{~s}, 1 \mathrm{H}, \operatorname{Ar}-\mathrm{H}), 6.60(\mathrm{~s}, 1 \mathrm{H}, \operatorname{Ar}-\mathrm{H})$, 4.47 (ddd, $1 \mathrm{H}, \mathrm{N}-\mathrm{HCH}), 3.87\left(\mathrm{~s}, 6 \mathrm{H}, 2 \times \mathrm{OCH}_{3}\right), 3.6-3.4$ (ddd, $1 \mathrm{H}, J=13.5,6.9,1.8 \mathrm{~Hz}, \mathrm{~N}-\mathrm{HCH}), 3.45(\mathrm{~d}, 1 \mathrm{H}$, $J=14.6 \mathrm{~Hz}, \alpha(\mathrm{CH}), 3.30(\mathrm{~d}, 1 \mathrm{H}, J=14.6 \mathrm{~Hz}, \alpha H \mathrm{HH})$, 3.06-2.82 (m, 2H, $\left.\mathrm{CH}_{2}\right) ; \delta_{\mathrm{C}}\left(\mathrm{CDCl}_{3}\right) 158.0,151.9(\mathrm{C}=\mathrm{O})$, $150.1,148.2\left(\mathrm{C}-\mathrm{OCH}_{3}\right), 131.4,125.5,124.8(\mathrm{C}), 91.0(\mathrm{C}-$ 1), $130.6(2), 128.7(2), 128.0,110.8,108.0(\mathrm{CH}), 56.0$ $\left(\mathrm{OCH}_{3}\right), 55.9\left(\mathrm{OCH}_{3}\right), 46.0,37.3,27.1\left(\mathrm{CH}_{2}\right) ; \mathrm{m} / \mathrm{z}(\%)$ $353\left(\mathrm{M}^{+}, 0.3\right), 280$ (100), 231 (35), 91 (32); Anal. Calcd for $\mathrm{C}_{20} \mathrm{H}_{19} \mathrm{NO}_{5}$ : C 67.98, H 5.42, N 3.96\%, found C 67.89, H $5.28, \mathrm{~N} 3.84$.

\section{Reaction of $N$-(2-phenylethyl)-3,4-dimethoxyphenyl- acetamide (7d)}

The yellow solid $(0.20 \mathrm{~g}, 23 \%)$ was identified as $9 d$. The residue from the filtrates was purified by column chromatography $\left(\mathrm{SiO}_{2}, \mathrm{CH}_{2} \mathrm{Cl}_{2} / \mathrm{CH}_{3} \mathrm{OH}\right.$ 20:0.5) to obtain $\mathbf{1 0}$ $(0.14 \mathrm{~g}, 16 \%$ yield $)$.

11,12-Dimethoxy-5,6-dihydroisoquino[1,2-b][3]benzazepine-8,9-dione (9d). Yellow crystals, $\mathrm{mp} 179-181^{\circ} \mathrm{C}$ $\left(\mathrm{CH}_{2} \mathrm{Cl}_{2}\right)$ (gas evolution); $\nu(\mathrm{KBr}) \quad \mathrm{cm}^{-1} 1645 ; \lambda_{\max }$ $(\mathrm{EtOH}) \mathrm{nm}(\log \epsilon): 398$ (2.85), 314 (2.96), 256 (4.01), $206(3.38) ; \delta_{\mathrm{H}}\left(\mathrm{CDCl}_{3}\right) 7.69(\mathrm{~s}, 1 \mathrm{H}, \mathrm{H}-10), 7.0-7.4(\mathrm{~m}$, $6 \mathrm{H}, \mathrm{ArH}$ ), 4.66 (br t, $\left.2 \mathrm{H}, J=6.9 \mathrm{~Hz}, \mathrm{H}-6,6^{\prime}\right), 4.09$ (s, 3H, $\left.\mathrm{OCH}_{3}\right), 4.00\left(\mathrm{~s}, 3 \mathrm{H}, \mathrm{OCH}_{3}\right), 3.21(\mathrm{br} \mathrm{t}, 2 \mathrm{H}, J=7.4 \mathrm{~Hz}, \mathrm{H}-$ $\left.5,5^{\prime}\right) ; \delta_{\mathrm{C}}\left(\mathrm{CDCl}_{3}\right)$ 163.6, 158.8, $(\mathrm{C}=\mathrm{O}), 152.6$ 152.4, $(C-$ $\left.\mathrm{OCH}_{3}\right), 142.1,140.1,135.4,117.8,107.7(\mathrm{C}), 129.2(2)$, 128.7, $127.8(2), 103.6,102.4(\mathrm{CH}), 54.2,35.5\left(\mathrm{CH}_{2}\right)$, 57.0, $56.5\left(\mathrm{OCH}_{3}\right) ; \mathrm{m} / \mathrm{z}(\%) 335\left(\mathrm{M}^{+}, 0\right), 307(10), 205$ (100); $\mathrm{m} / \mathrm{z}$ FAB-MS (\%) $336\left(\mathrm{MH}^{+}, 5\right)$; Anal. Calcd for $\mathrm{C}_{20} \mathrm{H}_{17} \mathrm{NO}_{4}$ : C 71.63, H 5.11, N 4.18\%, found $\mathrm{C} 71.69, \mathrm{H}$ 5.04, N 4.57.

2-\{1-[(Z)-(3,4-Dimethoxyphenyl)methylidene]-3,4-dihydro-2-isoquinolinyl\}-2-oxoacetic acid (10). Yellow needles, $\mathrm{mp} 162-163^{\circ} \mathrm{C}\left(\mathrm{CH}_{3} \mathrm{OH}\right) ; \nu(\mathrm{KBr}) \mathrm{cm}^{-1} 3300,1686,1685$; $\lambda_{\max }(\mathrm{EtOH}) \mathrm{nm}(\log \epsilon): 398$ (3.17), 254 (3.77), 210 (4.05); $\delta_{\mathrm{H}}\left(\mathrm{CDCl}_{3}\right) 7.66(\mathrm{~m}, 2 \mathrm{H}), 7.25(\mathrm{~m}, 4 \mathrm{H}), 6.90(\mathrm{~m}, 1 \mathrm{H}), 3.91$ $\left(\mathrm{s}, 3 \mathrm{H}, \mathrm{OCH}_{3}\right), 3.90\left(\mathrm{~s}, 3 \mathrm{H}, \mathrm{OCH}_{3}\right), 3.77\left(\mathrm{~m}, 2 \mathrm{H}, \mathrm{CH}_{2} \mathrm{~N}\right)$, $2.92\left(\mathrm{t}, 2 \mathrm{H}, J=8.0 \mathrm{~Hz}, \mathrm{CH}_{2} \mathrm{Ph}\right) ; \delta_{\mathrm{C}}\left(\mathrm{CDCl}_{3}\right) 170.8,167.4$ $(\mathrm{C}=\mathrm{O}), 149.2,148.7\left(\mathrm{C}-\mathrm{OCH}_{3}\right), 147.3(\mathrm{C}-4 \mathrm{a}), 137.8(\mathrm{C}-$ 1a), 128.8 (2), 128.6 (2), 126.7, $121.9(\mathrm{CH}), 121.5(\mathrm{C}-1)$, 111.1, 111.0, $(\mathrm{CH}), 107.9\left(\mathrm{C}-1^{\prime}\right), 55.8\left(\mathrm{OCH}_{3}\right), 52.8$ $\left(\mathrm{OCH}_{3}\right), 39.3(\mathrm{C}-3), 34.7(\mathrm{C}-4) ; \mathrm{m} / \mathrm{z}(\%) 353\left(\mathrm{M}^{+}, 100\right)$, 338 (1), 249 (17), 234 (12), 205 (54), 178 (14), 177 (67); 
Anal. Calcd for $\mathrm{C}_{20} \mathrm{H}_{19} \mathrm{NO}_{5}$ : C 67.98, H 5.42, N 3.96\%, found C 67.80, H 5.61, N 3.89.

\section{Reaction of 7 a with $(\mathrm{COCl})_{2} / \mathrm{SnCl}_{4}$ at $20^{\circ} \mathrm{C}$}

To a degassed (Ar) solution of the amide $7 \mathbf{a}(2.6 \mathrm{mmol})$ in dry dichloromethane $(25 \mathrm{~mL})$, in a septum sealed round bottom flask, oxalyl chloride $(6 \mathrm{~mL}, 26 \mathrm{mmol})$ was added. The solution was stirred at $20^{\circ} \mathrm{C}$ for $15 \mathrm{~min}$, followed by addition of stannyl chloride $(1.5 \mathrm{~mL}, 13 \mathrm{mmol})$ via a syringe. The mixture was stirred at $20^{\circ} \mathrm{C}$ for $24 \mathrm{~h}$. After this period, the reaction mixture was diluted with $\mathrm{CH}_{2} \mathrm{Cl}_{2}$ $(10 \mathrm{~mL})$ and a few drops of $1 \mathrm{M} \mathrm{HCl}$ were added to the solution. The dichloromethanic solution was washed with water, dried over $\mathrm{MgSO}_{4}$ and concentrated to dryness. The residue was separated by column chromatography $\left(\mathrm{SiO}_{2}\right.$, $\left.\mathrm{CH}_{2} \mathrm{Cl}_{2} / \mathrm{CH}_{3} \mathrm{OH} 20: 0.5\right)$ to obtain $8 \mathbf{a}(0.57 \mathrm{~g}, 63 \%)$.

10b-(3,4-Dimethoxybenzyl)-8,9-dimethoxy-6,10b-dihydro-5H-oxazolo[2,3-a]isoquinoline-2,3-dione (8a). White crystals, mp $160-161^{\circ} \mathrm{C}\left(\mathrm{CH}_{3} \mathrm{OH}\right) ; \nu(\mathrm{KBr}) \mathrm{cm}^{-1} 1803$, 1734; $\lambda_{\max }\left(\mathrm{CHCl}_{3}\right) \mathrm{nm}(\log \epsilon): 284$ (3.85), $246(2.44) ; \delta_{\mathrm{H}}$ $\left(\mathrm{CDCl}_{3}\right) 6.93(\mathrm{~s}, 1 \mathrm{H}), 6.76(\mathrm{~d}, 1 \mathrm{H}, J=8.7 \mathrm{~Hz}), 6.58(\mathrm{~m}, 3 \mathrm{H}$, $\mathrm{Ar}-\mathrm{H}), 4.45(\mathrm{~m}, 1 \mathrm{H}, \mathrm{N}-\mathrm{HCH}), 3.90\left(\mathrm{~s}, 3 \mathrm{H}, \mathrm{OCH}_{3}\right), 3.87$ (s, $\left.3 \mathrm{H}, \mathrm{OCH}_{3}\right), 3.83\left(\mathrm{~s}, 3 \mathrm{H}, \mathrm{OCH}_{3}\right), 3.81\left(\mathrm{~s}, 3 \mathrm{H}, \mathrm{OCH}_{3}\right), 3.45$ (d, $1 \mathrm{H}, J=14.8 \mathrm{~Hz}, \alpha H C H), 3.23(\mathrm{~d}, 1 \mathrm{H}, J=14.8 \mathrm{~Hz}, \alpha \mathrm{HCH})$, 3.5-3.1 (m, 2H, CH $), 2.8(\mathrm{~m}, 1 \mathrm{H}, \mathrm{N}-\mathrm{HCH}) ; \delta_{\mathrm{C}}\left(\mathrm{CDCl}_{3}\right)$ $158.2,151.9(\mathrm{C}=\mathrm{O}), 150.2,149.04,149.01,148.4(C-$ $\left.\mathrm{OCH}_{3}\right), 125.7,124.9,123.8$ (C), 123.0, 113.7, 111.2, 111.0, $108.1(\mathrm{CH}), \quad 91.2(\mathrm{C}-1), \quad 56.2\left(\mathrm{OCH}_{3}\right), \quad 56.0$ $\left(2 \times \mathrm{OCH}_{3}\right), 55.8\left(\mathrm{OCH}_{3}\right), 45.9,37.3,27.2\left(\mathrm{CH}_{2}\right) ; \mathrm{m} / \mathrm{z}(\%)$ $413\left(\mathrm{M}^{+}, 2\right), 341$ (63), 340 (100), 326 (37), 310 (43), 234 (47), 151 (62); Anal. Calcd for $\mathrm{C}_{22} \mathrm{H}_{23} \mathrm{NO}_{7} \cdot 1 \mathrm{H}_{2} \mathrm{O}$ : $\mathrm{C} 61.23$, H 5.84, N 3.25\%, found C 61.25, H 5.49, N 3.37 .

\section{Reaction of amides with $(\mathrm{COCl})_{2} / \mathrm{SnCl}_{4}$ at $60^{\circ} \mathrm{C}$. General procedure}

To a degassed (Ar) solution of the amide $7 \mathbf{a}-\mathbf{c}(2.6 \mathrm{mmol})$ in dry dichloromethane $(25 \mathrm{~mL})$, in a septum sealed round bottom flask, oxalyl chloride $(6 \mathrm{~mL}, 26 \mathrm{mmol})$ was added. The solution was stirred at $20^{\circ} \mathrm{C}$ for $15 \mathrm{~min}$, and the tin(IV) chloride ( $1.5 \mathrm{~mL}, 13 \mathrm{mmol})$ added via a syringe. The mixture was stirred at $60^{\circ} \mathrm{C}$ for $24 \mathrm{~h}$, time during which a solid precipitated. The yellow solid was filtered, washed with $\mathrm{CH}_{2} \mathrm{Cl}_{2}$, treated with $1 \mathrm{M} \mathrm{NaOH}(25 \mathrm{~mL})$ for $30 \mathrm{~min}$ and then filtered again to give $\mathbf{9 a}-\mathbf{c}$. The organic phase was washed with water, dried over $\mathrm{MgSO}_{4}$, concentrated in vacuo and separated by column chromatography to obtain the stated products.

\section{Reaction of amide $7 \mathbf{a}$}

9a: Yellow solid, $0.3 \mathrm{~g}, 29 \%$. The yellow solid before treatment with $1 \mathrm{M} \mathrm{NaOH}$ was characterized as $\mathbf{9 a} \cdot \mathbf{S n C l}_{\mathbf{2}}: 1.5 \mathrm{~g}$, $99 \%$; mp $>300^{\circ} \mathrm{C}$. Anal. Calcd for $\mathrm{C}_{22} \mathrm{H}_{21} \mathrm{NO}_{6} \cdot \mathrm{SnCl}_{2}$ : C 45.13, H 3.62, N 2.39\%, found C 45.20, H 3.78, N 2.41.

\section{Reaction of amide $7 \mathrm{~b}$}

9b: Yellow solid, $0.3 \mathrm{~g}, 27 \%$. The residue from the filtrates was separated by column chromatography $\left(\mathrm{SiO}_{2}, \mathrm{CH}_{2} \mathrm{Cl}_{2}\right.$ and $\mathrm{CH}_{2} \mathrm{Cl}_{2} / \mathrm{CH}_{3} \mathrm{OH}$ 20:0.5) to obtain a 1:1 mixture of $\mathbf{8 b}$ and 11. The solid mixture was crystallized from $\mathrm{CH}_{2} \mathrm{Cl}_{2} /$ $\mathrm{CH}_{3} \mathrm{OH}$, the crystals were washed several times with methanol to obtain pure $\mathbf{8 b}(0.09 \mathrm{~g}, 9 \%)$, and the mother liquor was used to isolate $11(0.09 \mathrm{~g}, 10 \%)$ by recrystallization from $\mathrm{CH}_{3} \mathrm{OH}$.

10b-(4-Methoxybenzyl)-8,9-dimethoxy-6,10b-dihydro-5 $H$ oxazolo[2,3-a]isoquinoline-2,3-dione (8b). Colourless needles, mp $147-150^{\circ} \mathrm{C}\left(\mathrm{CHCl}_{3} / \mathrm{CH}_{3} \mathrm{OH}\right)$ (gas evolution); $\nu(\mathrm{KBr}) \mathrm{cm}^{-1} 1801,1734,1610 ; \lambda_{\max }\left(\mathrm{CHCl}_{3}\right) \mathrm{nm}(\log \epsilon)$ : 284 (3.72), 246 (3.99); $\delta_{\mathrm{H}}\left(\mathrm{CDCl}_{3}\right) 7.00(\mathrm{~d}, 2 \mathrm{H}, J=8.6 \mathrm{~Hz}$, Ar-H), 6.90 (s, 1H, Ar-H), 6.80 (d, 2H, J=8.6 Hz, Ar-H), 6.60 (s, 1H, Ar-H), 4.48 (m, 1H, $\left.\mathrm{NCH}_{2}\right), 3.89$ (s, 3H, $\mathrm{OCH}_{3}$ ), $3.87\left(\mathrm{~s}, 3 \mathrm{H}, \mathrm{OCH}_{3}\right), 3.75\left(\mathrm{~s}, 3 \mathrm{H}, \mathrm{OCH}_{3}\right), 3.45(\mathrm{~d}, 1 \mathrm{H}$, $J=14.8 \mathrm{~Hz}, \alpha(\mathrm{CH}), 3.60-3.35\left(\mathrm{~m}, 1 \mathrm{H}, \mathrm{NCH}_{2}\right), 3.23(\mathrm{~d}$, $1 \mathrm{H}, J=14.8 \mathrm{~Hz}, \alpha H \mathrm{CH}), 3.2-3.0\left(\mathrm{~m}, 1 \mathrm{H}, \mathrm{CH}_{2}\right), 2.70(\mathrm{~m}$, $\left.1 \mathrm{H}, \mathrm{CH}_{2}\right) ; \delta_{\mathrm{C}}\left(\mathrm{CDCl}_{3}\right)$ 159.4, $158.2(\mathrm{C}=\mathrm{O}), 152.0,150.2$, $148.3\left(\mathrm{C}-\mathrm{OCH}_{3}\right), 125.6,124.8,123.2(\mathrm{C}), 91.1(\mathrm{C}-1), 131.8$ (2), 114.2 (2), 110.9, $108.1(\mathrm{CH}), 56.1\left(\mathrm{OCH}_{3}\right), 56.0$ $\left(\mathrm{OCH}_{3}\right), 55.2\left(\mathrm{OCH}_{3}\right), 45.4,37.3,27.3\left(\mathrm{CH}_{2}\right) ; \mathrm{m} / \mathrm{z}(\%)$ $383\left(\mathrm{M}^{+}, 1\right), 310$ (100), 280 (41), 234 (29), 121 (78); Anal. Calcd for $\mathrm{C}_{21} \mathrm{H}_{21} \mathrm{NO}_{6}$ : C $65.79, \mathrm{H} 5.52, \mathrm{~N} 3.65 \%$, found C 65.67, H 5.59, N 3.69.

5,6-Dihydro-8,9-dimethoxy-1-(4-methoxyphenyl)-pyrrolo[2,3-a]isoquinoline-2,3-dione (11). Red needles, mp 212$215^{\circ} \mathrm{C}$ (recrystallized from $\left.\mathrm{CH}_{3} \mathrm{OH}\right) ; \nu(\mathrm{KBr}) \mathrm{cm}^{-1} 1740$, 1679; $\lambda_{\max }\left(\mathrm{CHCl}_{3}\right) \mathrm{nm}(\log \epsilon): 512$ (3.55), 460 (3.57), 444 (3.58), 380 (3.88), 314 (3.91), 258 (4.07), $246(4.09) ; \delta_{\mathrm{H}}$ $\left(\mathrm{CDCl}_{3}\right) 7.26(\mathrm{~d}, 2 \mathrm{H}, J=8.7 \mathrm{~Hz}, \mathrm{Ar}-\mathrm{H}), 7.03$ (s, 1H, Ar$\mathrm{H}), 6.92(\mathrm{~d}, 2 \mathrm{H}, J=8.7 \mathrm{~Hz}, \operatorname{Ar}-\mathrm{H}), 6.73$ (s, 1H, Ar-H), 3.9-3.7 (br t, $\left.2 \mathrm{H}, \mathrm{NCH}_{2}\right), 3.93\left(\mathrm{~s}, 3 \mathrm{H}, \mathrm{OCH}_{3}\right), 3.80(\mathrm{~s}$, $\left.3 \mathrm{H}, \mathrm{OCH}_{3}\right), 3.37\left(\mathrm{~s}, 3 \mathrm{H}, \mathrm{OCH}_{3}\right), 3.05(\mathrm{t}, 2 \mathrm{H}, J=6.1 \mathrm{~Hz}$, $\left.\mathrm{CH}_{2}\right) ; \quad \delta_{\mathrm{C}}\left(\mathrm{CDCl}_{3}\right)$ 159.3, $158.2(\mathrm{C}=\mathrm{O}), 156.7,153.3$, $147.8\left(\mathrm{C}-\mathrm{OCH}_{3}\right), 132.8,122.3,116.8,108.1(\mathrm{C}), 131.3$ (2), $114.3(2), 111.9,111.1(\mathrm{CH}), 56.2\left(\mathrm{OCH}_{3}\right), 55.4$ $\left(2 \times \mathrm{OCH}_{3}\right), 36.3\left(\mathrm{NCH}_{2}\right), 28.6\left(\mathrm{CH}_{2}\right) ; \mathrm{m} / \mathrm{z}(\%) 365\left(\mathrm{M}^{+}\right.$, 53), 336 (100); Anal. Calcd for $\mathrm{C}_{21} \mathrm{H}_{19} \mathrm{NO}_{5}$ : C 69.03, $\mathrm{H}$ 5.24, N 3.83\%, found C 68.70, H 5.11, N 4.02.

\section{Reaction of amide 7c}

9c: Yellow solid, $0.17 \mathrm{~g}, 21 \%$. From column chromatography $\left(\mathrm{SiO}_{2}, \mathrm{CH}_{2} \mathrm{Cl}_{2}\right.$ and $\mathrm{CH}_{2} \mathrm{Cl}_{2} / \mathrm{CH}_{3} \mathrm{OH}$ 20:0.5) of the residue from the filtrates $8 \mathbf{c}$ (white solid, $0.39 \mathrm{~g}, 42 \%$ ) was obtained.

\section{Decarbonylation of 9a,b}

A mixture of $9 \mathbf{a}, \mathbf{b}(0.3 \mathrm{mmol}), \mathrm{NaOH}(1.5 \mathrm{~g})$ and methanol $(40 \mathrm{~mL})$ was refluxed for $48 \mathrm{~h}$. The solvent was removed in vacuo, and the reaction crude was acidified with concentrated $\mathrm{HCl}(\mathrm{pH} 5)$. The mixture was diluted with water $(15 \mathrm{~mL})$ and extracted with chloroform $(2 \times 25 \mathrm{~mL})$. The organic layer was washed with water $(15 \mathrm{~mL})$, dried over $\mathrm{MgSO}_{4}$ and concentrated in vacuo. The residue was purified by column chromatography $\left(\mathrm{SiO}_{2}, \mathrm{CH}_{2} \mathrm{Cl}_{2}\right)$ to give $\mathbf{1 a}, \mathbf{b}$.

8-Oxopseudopalmatine (1a). Orange-yellow solid, yield: $0.06 \mathrm{~g}, 66 \%$; mp $185-186^{\circ} \mathrm{C}\left(\mathrm{CH}_{3} \mathrm{OH}\right)\left(\mathrm{mp} 198-199^{\circ} \mathrm{C}\right.$, $\left.187-188^{\circ} \mathrm{C}\right) ;^{1 \mathrm{c}} \delta_{\mathrm{H}}\left(\mathrm{CDCl}_{3}\right) 7.79(\mathrm{~s}, 1 \mathrm{H}, \mathrm{Ar}-\mathrm{H}), 7.23(\mathrm{~s}$, $1 \mathrm{H}, \mathrm{Ar}-\mathrm{H}), 6.93$ (s, 1H, Ar-H), 6.82 (s, 1H, Ar-H), 6.73 (s, $1 \mathrm{H}, \mathrm{Ar}-\mathrm{H}), 4.35\left(\mathrm{t}, 2 \mathrm{H}, J=6.0 \mathrm{~Hz}, \mathrm{NCH}_{2}\right), 3.97(\mathrm{~s}, 6 \mathrm{H}$, 
$\left.2 \times \mathrm{OCH}_{3}\right), 3.93\left(\mathrm{~s}, 6 \mathrm{H}, 2 \times \mathrm{OCH}_{3}\right), 2.93(\mathrm{t}, 2 \mathrm{H}, J=6.0 \mathrm{~Hz}$, $\left.\mathrm{CH}_{2}\right) ; \quad \delta_{\mathrm{C}}\left(\mathrm{CDCl}_{3}\right) 161.3(\mathrm{C}=\mathrm{O}), 153.4,150.0,148.9$, $148.4\left(C-\mathrm{OCH}_{3}\right), 136.1,132.1,128.3,122.4,118.5(\mathrm{C})$, $110.5,107.8,107.6,105.9,101.0(\mathrm{CH}), 56.1\left(2 \times \mathrm{OCH}_{3}\right)$, $56.0\left(2 \times \mathrm{OCH}_{3}\right), 39.7\left(\mathrm{NCH}_{2}\right), 28.1\left(\mathrm{CH}_{2}\right) ; \mathrm{m} / \mathrm{z}(\%) 367$ $\left(\mathrm{M}^{+}, 100\right), 352$ (60). This compound (1a) was found to be identical (TLC, MS, ${ }^{1} \mathrm{H}$ and ${ }^{13} \mathrm{C} \mathrm{NMR}$ ) with an authentic sample obtained by $\mathrm{K}_{3}\left[\mathrm{Fe}(\mathrm{CN})_{6}\right]$ oxidation of synthetic pseudopalmatine chloride. ${ }^{1 \mathrm{~b}}$

2,3,10-Trimethoxy-5,6-dihydro-8H-isoquino[3,2-a]isoquinolin-8-one (1b). Yellowish syrup, $0.05 \mathrm{~g}, 50 \%$; $\nu$ $(\mathrm{NaCl}) \mathrm{cm}^{-1} 1685 ; \lambda_{\max }\left(\mathrm{CHCl}_{3}\right) \mathrm{nm}(\log \epsilon): 378 \mathrm{sh}$ (3.69), $362 \mathrm{sh}$ (3.90), $340(4.11), 246(3.97) ; \delta_{\mathrm{H}}\left(\mathrm{CDCl}_{3}\right)$ 7.81 (d, 1H, $J=2.6 \mathrm{~Hz}, \mathrm{H}-9), 7.48$ (d, $1 \mathrm{H}, J=8.7 \mathrm{~Hz}, \mathrm{H}-12)$, 7.26-7.21 (m, 2H, Ar-H), 6.85 (s, 1H, Ar-H), 6.73 (s, 1H, $\mathrm{Ar}-\mathrm{H}), 4.36$ (t, 2H, J=6.2 Hz, $\left.\mathrm{NCH}_{2}\right), 3.97$ (s, 3H, $\mathrm{OCH}_{3}$ ), $3.92\left(\mathrm{~s}, 6 \mathrm{H}, 2 \times \mathrm{OCH}_{3}\right), 2.93\left(\mathrm{t}, 2 \mathrm{H}, J=6.1 \mathrm{~Hz}, \mathrm{CH}_{2}\right) ; \delta_{\mathrm{C}}$ $\left(\mathrm{CDCl}_{3}\right) 161.8(\mathrm{C}=\mathrm{O}), 158.4(\mathrm{C}-10), 150.1,148.5(\mathrm{C}-2$, C-3), 135.3, 130.9, 128.3, 125.7, 122.6 (C), 127.6, 123.1, $110.5,107.70,107.66,101.4(\mathrm{CH}), 56.3\left(\mathrm{OCH}_{3}\right), 56.0$ $\left(\mathrm{OCH}_{3}\right), 55.7\left(\mathrm{OCH}_{3}\right), 39.9\left(\mathrm{NCH}_{2}\right), 28.1\left(\mathrm{CH}_{2}\right) ; \mathrm{m} / \mathrm{z}(\%)$ $337\left(\mathrm{M}^{+}, 100\right), 322$ (60); Anal. Calcd for $\mathrm{C}_{20} \mathrm{H}_{19} \mathrm{NO}_{4}$ : $\mathrm{C}$ $71.20, \mathrm{H} 5.68, \mathrm{~N} 4.15 \%$, found C 71.00, H 5.62, N 4.02 .

\section{Thermal decarbonylation of $9 a, b$}

The net products $9 \mathbf{a}, \mathbf{b}(1 \mathrm{mmol})$ were heated at 175 and $160^{\circ} \mathrm{C}$, respectively, until gas evolution ceased (ca. $1 \mathrm{~h}$ ). After this period, the reaction was cooled and the crude residue was separated by column chromatography $\left(\mathrm{SiO}_{2}\right.$, $\mathrm{CH}_{2} \mathrm{Cl}_{2}$ ) to obtain $\mathbf{1 a}, \mathbf{b}$ in 70 and $36 \%$ yield, respectively.

\section{Acknowledgements}

We thank Dr E. Rodríguez-Castellón for the recording XPS spectra and Dr F. J. Alonso for the cytotoxicity analyses. This work was financially supported by Spain's DGES (Project PB97/1077).

\section{References}

1. (a) Bhakuni, D. S.; Jain, S. Protoberberine Alkaloids. In The Alkaloids; Brossi, A. Ed.; Academic Press: New York, 1986; Vol. 28, Chapter 2. (b) Shamma, M. The Protoberberines and Retroprotoberberines. In The Isoquinoline Alkaloids. Chemistry and Pharmacology; Blomquist, A. T.; Wasserman, H., Eds.; Academic
Press: New York, 1972; Vol. 25, p. 268. (c) Southon, I. W.; Buckingham, J. Dictionary of Alkaloids; Chapman and Hall: London, 1989. (d) Bentley, K. W. Berberines and Tetrahydroberberines. In The Isoquinoline Alkaloids; Ravindranath, B., Ed.; Harwood Academic: Bangalore, 1998; Vol. 1, p. 219.

2. González, M. C.; Zafra-Polo, M. C.; Amparo-Blázquez, M.; Serrano, A.; Cortés, D. J. Nat. Prod. 1997, 60, 108.

3. Schemeller, T.; Latzbruning, B.; Wink, M. Nat. Med. 1997, 51, 516.

4. Chi, J. F.; Chu, S. H.; Lee, C. S.; Su, M. J. Can. J. Cardiol. 1997, 13, 1103.

5. (a) Shamma, M.; Rahimizadeh, M. J. Nat. Prod. 1986, 49, 398. (b) Rahimizadeh, M. J. Sci. Islamic Repub. Iran 1996, 7, 172.

6. Hanaoka, M. Transformation Reactions of Protoberberine Alkaloids. In The Alkaloids, Brossi, A., Ed.; Academic Press: New York, 1988; Vol. 33 (Chapter 3).

7. D'Sa, A. S.; Deodhar, K. D. Indian J. Chem. B 1980, 19, 999. 8. (a) Weimar, C.; Angerer, S. V.; Wiegrebe, W. Arch. Pharm. (Weinheim) 1991, 324, 509. (b) Naito, T.; Katsumi, K.; Tada, Y.; Ninomiya, I. Heterocycles 1983, 20, 779.

9. Grigg, R.; Sridharan, V.; Stevenson, P.; Sukirthalingam, S.; Worakun, T. Tetrahedron 1990, 46, 4003.

10. Yang, N. C.; Shani, A.; Lenz, G. R. J. Am. Chem. Soc. 1966, $88,5369$.

11. Cobas, A.; Guitian, E.; Castedo, L. J. Org. Chem. 1992, 57, 6765 .

12. MacLean, D. B. Stud. Org. Chem. (Amsterdam) 1985, 20, 113. 13. Haimova, M. A.; Ognyanov, V. I.; Mollov, N. M. Synthesis 1980, 845.

14. Kessar, S. V.; Singh, P.; Vohra, R.; Kaur, N. P.; Venugopal, D. J. Org. Chem. 1992, 57, 6716.

15. Reimann, E.; Benend, H. Monatsh. Chem. 1992, 123, 939.

16. Kenkov, A. P.; Ivanov, I. L. Tetrahedron 1996, 52, 12299.

17. Lamas, C.; Saá, C.; Castedo, L.; Domínguez, D. Tetrahedron Lett. 1992, 33, 5653.

18. (a) Suau, R.; López-Romero, J. M.; Rico, R. Tetrahedron Lett. 1996, 37, 9357. (b) Suau, R.; López-Romero, J. M.; Rico, R. Tetrahedron 1997, 53, 14397.

19. Larsen, R. D.; Reamer, R. A.; Corley, G.; Davis, P.; Grabowski, E. J. J.; Reider, P. J.; Shinkai, I. J. Org. Chem. 1991, 56,6034 .

20. Bryon Gill, G. Benzyl-Benzylic Acid Rearrengements. In Comprehensive Organic Synthesis, Trost, B. M., Fleming, I., Pattenden, G., Eds.; Pergamon: New York, 1991; Vol. 3, p 821 (Chapter 6).

21. (a) Suau, R.; López-Romero, J. M.; Rico, R.; Alonso, F. J.; Lobo, C. Tetrahedron 1996, 52, 11307. (b) Mosmann, T. Immunol. Meth. 1983, 65, 55. 